\title{
An ion cooler-buncher for high-sensitivity collinear laser spectroscopy at ISOLDE
}

\author{
E. Mané ${ }^{1, a}$, J. Billowes ${ }^{1}$, K. Blaum ${ }^{2}$, P. Campbell ${ }^{1}$, B. Cheal ${ }^{1}$, P. Delahaye ${ }^{3}$, K.T. Flanagan ${ }^{4}$, D.H. Forest ${ }^{5}$, \\ H. Franberg ${ }^{3}$, C. Geppert ${ }^{6}$, T. Giles ${ }^{3}$, A. Jokinen ${ }^{7,8}$, M. Kowalska ${ }^{3}$, R. Neugart ${ }^{6}$, G. Neyens ${ }^{9}$, W. Nörtershäuser ${ }^{6}$, \\ I. Podadera ${ }^{10}$, G. Tungate ${ }^{5}$, P. Vingerhoets ${ }^{9}$, and D.T. Yordanov ${ }^{2}$ \\ 1 The University of Manchester, Manchester M13 9PL, UK \\ 2 Max-Planck-Institute for Nuclear Physics, 69117 Heidelberg, Germany \\ 3 ISOLDE-CERN, route de Meyrin, CH-1211 Geneva 23, Switzerland \\ 4 IPN, IN2P3-CNRS, F-91406 Orsay Cedex, France \\ 5 The University of Birmingham, Birmingham B15 2TT, UK \\ 6 Institute for Physics, Johannes Gutenberg University, 55099 Mainz, Germany \\ 7 Department of Physics, University of Jyväskylä, P.O. Box 35 (YFL), FIN-40014, Finland \\ 8 Helsinki Institute of Physics, University of Helsinki, P.O. Box 64, FIN-00014, Finland \\ 9 K.U. Leuven, IKS, Celestijnenlaan 200 D, B-3001 Leuven, Belgium \\ 10 Accelerator Group, CIEMAT, Avda. Complutense 22, 28040 Madrid, Spain
}

Received: 27 November 2008 / Revised: 15 May 2009

Published online: 16 June 2009 - (c) Società Italiana di Fisica / Springer-Verlag 2009

Communicated by J. Äystö

\begin{abstract}
A gas-filled segmented linear Paul trap has been installed at the focal plane of the high-resolution separator (HRS) at CERN-ISOLDE. As well as providing beams with a reduced transverse emittance, this device is also able to accumulate the ions and release the sample in bunches with a well-defined time structure. This has recently permitted collinear laser spectroscopy with stable and radioactive bunched beams to be demonstrated at ISOLDE. Surface-ionized ${ }^{39,44,46} \mathrm{~K}$ and ${ }^{85} \mathrm{Rb}$ beams were accelerated to $30 \mathrm{keV}$, mass separated and injected into the trap for subsequent extraction and delivery to the laser setup. The ions were neutralized in a charge exchange cell and excited with a co-propagating laser. The small ion beam emittance allowed focussing in the ion-laser overlap region, which is essential to achieve the best experimental sensitivity. Fluorescent photons were detected by a photomultiplier tube as a frequency scan was taken. A gate (typically $7-12 \mu$ s wide) was set on the photomultiplier signal to accept the fluorescent photons within the time window defined by the bunch. Thus, using accumulation times of $100 \mathrm{~ms}$, the dominant contribution to background due to continuous laser scattering could be reduced by a factor of up to $4 \times 10^{4}$.
\end{abstract}

PACS. 37.10.Rs Ion cooling - 37.10.Ty Ion trapping - 42.62.Fi Laser spectroscopy - 31.30.Gs Hyperfine interactions and isotope effects

\section{Introduction}

High-resolution collinear laser spectroscopy with fast ion or atom beams is a well-established technique for the determination of nuclear moments, spins and changes in the mean square charge radii of radioisotopes $[1,2]$. The most recent compilation with the status of the measurements of these observables determined by optical methods can be found in [3]. However, the sensitivity of the collinear laser technique with laser-induced fluorescence detection is limited by the background originating from detector dark counts and laser scatter in the light collection region from

\footnotetext{
${ }^{a}$ e-mail: Ernesto@cern.ch
}

apertures and other components in the beam line. Taken together, these sources of background can have a detrimental effect on the fluorescence measurements of ions produced with low fluxes (below $10^{6}$ ions/s) at the point of interaction. Trapping the ion beam in a linear Paul trap, and releasing the sample in bunches with a well-defined time structure provides a way to suppress the background from the laser scatter, and therefore enhance the signalto-noise ratio, as pioneered in [4].

\section{The ISOLDE ion cooler-buncher}

Gas-filled linear Paul traps have become commonplace in radioactive ion beam facilities around the world. This is 


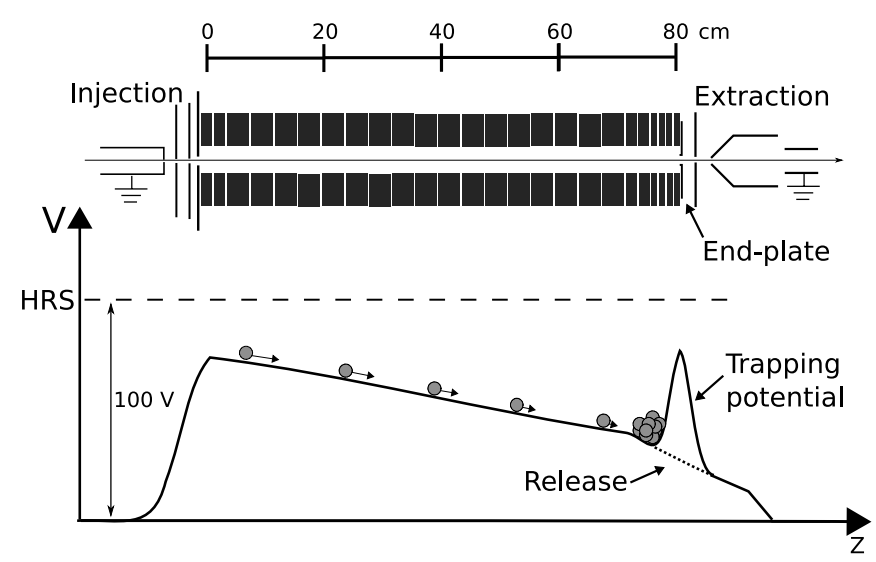

Fig. 1. Diagram of the segmented radiofrequency quadrupole trap.

due to the versatility and universality of these devices, which provide beams of low emittance and the option of a pulsed release of ions. Examples of facilities which have such an apparatus in operation include ISOLTRAP at ISOLDE [5], the IGISOL facility in Jyväskylä [6] and LEBIT at MSU [7].

At ISOLDE, a general-purpose linear Paul trap, ISCOOL, has recently been developed and commissioned for operation at the focal plane of the high-resolution separator (HRS). The purpose of the device is to deliver ion beams with an expected transverse emittance of less than $3 \pi \mathrm{mm} \cdot \operatorname{mrad}$ at $60 \mathrm{keV}$ and a low-energy spread $(<1 \mathrm{eV})$, either continuously or in bunches with a well-defined temporal structure.

The device consists of injection electrodes, a quadrupole structure for trapping the ions in the transverse plane, and extraction electrodes. The trap is realized with four rods coupled pairwise. The applied voltage to a pair of electrodes is $V_{r f} \cos (\Omega t)$. The same voltage is applied to the alternate pair, but with an opposing polarity. The radiofrequency field applied to the quadrupole is used to confine the ion cloud in the radial direction. The rods are surrounded by 25 segmented DC electrodes. The structure is maintained typically $100 \mathrm{~V}$ below the high voltage of the HRS. The gas, helium, fills the quadrupole volume. A pressure of about $0.1 \mathrm{mbar}$ is used to slow and cool the ions via thermal collisions. A differential pumping system is used to keep the pressure on either side of the device below $10^{-7}$ mbar. The DC field, which is independently applied to each segment permits the creation of a potential gradient of $0.2 \mathrm{~V} / \mathrm{cm}$ in order to guide the ions to the trap exit. The ions can be extracted as a continuous flux or they can be accumulated and released in short bunches, as shown by fig. 1 .

A more detailed description of this device together with its design specifications can be found in $[8,9]$. Prior to the on-line commissioning phase, off-line tests were undertaken with ISCOOL in a dedicated test bench, under conditions similar to that found at the HRS. The results of these tests, which investigated the transmission in continuous mode as well as the extracted emittance at $30 \mathrm{keV}$ for a surface alkali ion source are reported in [10]. This

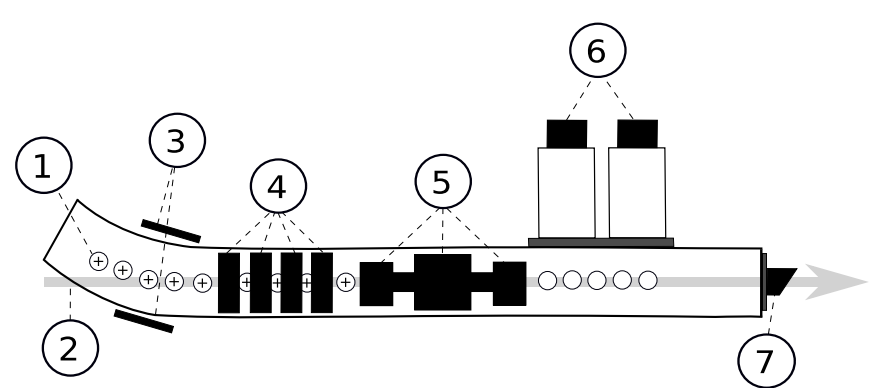

Fig. 2. Collinear laser spectroscopy setup (COLLAPS) at ISOLDE. 1) Single charged ions; 2) laser beam; 3) electrostatic deflection plates; 4) post-acceleration electrodes; 5) charge exchange cell (CEC); 6) photomultiplier tubes; 7) brewster window.

work reports on the first use of ISCOOL for collinear laser spectroscopy with fast beams at ISOLDE.

\section{Collinear laser spectroscopy of ${ }^{39,44,46} \mathrm{~K}$ and ${ }^{85} \mathrm{Rb}$}

Tests were made on stable ${ }^{39} \mathrm{~K}$ and on radioactive ${ }^{44,46} \mathrm{~K}$, produced from a tantalum HRS target. In addition, stable ${ }^{85} \mathrm{Rb}$ was studied. For the potassium ions, ISCOOL operated with rf amplitude $V_{r f}=280 \mathrm{~V}$ and $\Omega=520 \mathrm{kHz}$, whereas for rubidium, $V_{r f}=270 \mathrm{~V}$ and $\Omega=450 \mathrm{kHz}$. The ion beam from ISCOOL were steered to the collinear laser spectroscopy beam line, COLLAPS [11-13]. Figure 2 shows a simplified scheme of the setup. The ions were neutralized by passage through a charge exchange cell filled with hot potassium vapour, which was placed before the light collection region. The neutral beam was overlapped with a collinear Ti:Sa laser beam, co-propagating with the ion beam direction. The high velocity of the atom beam acted to compress the forward velocity spread, which allowed high-resolution spectroscopy to be performed. A tuning potential on the charge exchange cell was applied to Doppler shift the laser light (in the rest frame to the atom) into resonance. The transitions chosen were the $D_{2}$ lines both for potassium $(766 \mathrm{~nm})$ and rubidium $(780 \mathrm{~nm})$. The atoms in the interaction region were resonantly excited with the laser and the subsequent fluorescence photons were counted with two red-sensitive photomultiplier tubes as the tuning voltage was scanned.

\section{Laser and ion beam overlap}

Two removable apertures were placed in the COLLAPS beam line to tune the ion beam. A $1 \mathrm{~mm}$ diameter aperture was used to maximise the overlap of the ion and laser beams in the vicinity of the photon detection region. The narrow waist minimised the laser power required, with a commensurate reduction of the scattered laser light. A second aperture, with a $4 \mathrm{~mm}$ diameter and placed downstream the detection region, was used to ensure a slow 
focussing of the ion beam. During the off-line commissioning of ISCOOL, the emittance of an alkali surface ion source was reduced from $35 \pi \mathrm{mm} \cdot \operatorname{mrad}$ to $3 \pi \mathrm{mm} \cdot \mathrm{mrad}$ at $30 \mathrm{keV}$ [10]. This allows the focussing of the ion beam into the interaction region of the COLLAPS beam line to be improved, providing a better laser-ion overlap.

A Faraday cup was placed at the end of the beam line in order to monitor the beam current. For ${ }^{39} \mathrm{~K}, 2.2 \mathrm{nA}$ was measured without the apertures. Placing the $1 \mathrm{~mm}$ diameter aperture, $45 \%$ went through. With both apertures in, a third of the beam was transmitted. The last element of the setup was a Brewster window, which served to let the laser light through without too much internal reflection and also helped with the laser alignment.

\section{Data acquisition and timing of signals}

The ion beam energy was defined by the ISCOOL platform voltage. For these tests, the ions were accelerated up to $30 \mathrm{keV}$. Doppler-tuning was performed by postaccelerating the ions prior to the neutralization region. A post-acceleration voltage range $\Delta U_{D}$ was defined for a given scanning region of the hyperfine structure. This region was divided into $N$ "channels", and each channel was set to count photons for a fixed dwell time, which is typically $100 \mathrm{~ms}$. The system advanced to the next channel upon receiving a fresh bunch signal, whilst the next ion bunch was being accumulated in the trap. This timing was controlled by a function generator, which was used to generate a TTL signal in order to fast-switch between one power supply set to $50 \mathrm{~V}$ and another to $0 \mathrm{~V}$. The switch output was connected to the end plate electrode of the ion trap. By varying the period time in the function generator, it was possible to control the accumulation and release time of the bunches. The same TTL signal which was sent to open the trap, was used to generate a gate signal. A delay was introduced to account for the time the ions were transported and neutralized in the CEC. The resonant photons were counted in coincidence with the arrival of the ion bunch at the light collection region, thereby vetoing any background event which happened outside this time window.

\section{Optimal bunch width}

In order to observe the temporal structure of the ion bunch, and hence determine the optimal bunch width, a multichannel scaler (MCS) was used to count the photons while the ions were held in resonance. The MCS was triggered by the same TTL pulse which was used to open the end plate in the trapping region. Of special importance was to understand the effect of the accumulation times on the bunch width. Figure 3 summarises the measurements carried out for ${ }^{85} \mathrm{Rb}$ ions against different accumulation times, ranging from $2.5 \mathrm{~ms}$ to $100 \mathrm{~ms}$. The amount of ions injected into the trap could be reduced by changing the offset position of a slit placed after the focal plane of the

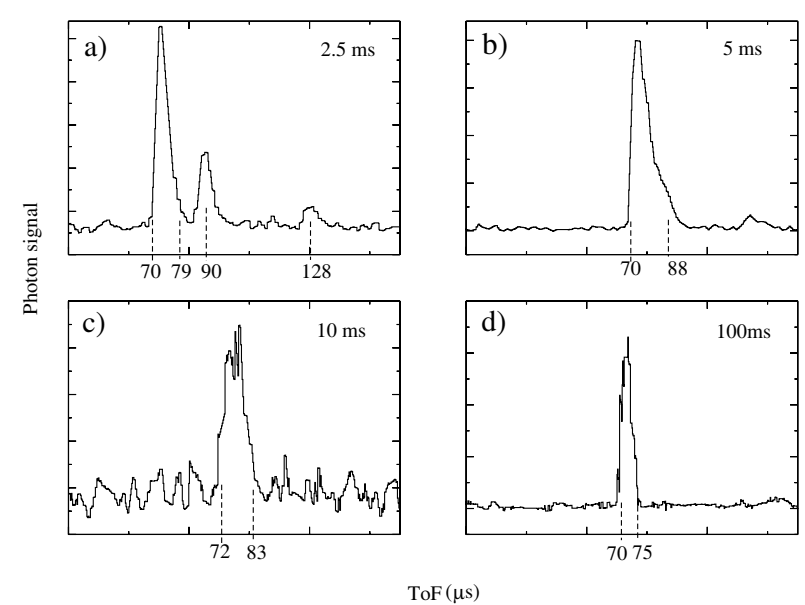

Fig. 3. Bunch width of ${ }^{85} \mathrm{Rb}$ from the photon arrival times. The time markers are in $\mu \mathrm{s}$. In (a) and (b) the number of bunched ions are $3 \times 10^{4}$ and $6 \times 10^{4}$, whereas in (c) and (d), $5 \times 10^{3}$ and $5 \times 10^{4}$ ions were bunched.

HRS. Figures 3(a) and (b) show how the bunches vary for different accumulation times, but with the ions injected into the trap kept at the same rate. In fig. 3(a), there are $3 \times 10^{4}$ ions in the bunch, whereas in fig. $3(\mathrm{~b})$, there are twice as much. Both upper figures show a structure in the bunches, but the data indicates that a twofold increase in the number of ions in the trap caused some of the structure to merge. The origin of this structure is not understood. A similar study was performed with longer accumulation times, but with the ion current reduced to avoid space-charge effects. By using the fluorescence detection efficiency, the number of bunched ions is estimated to be $5 \times 10^{3}$ in fig. $3(\mathrm{c})$, and an order of magnitude higher in fig. 3(d). The number of ions per bunch in fig. 3(d) is similar to that used for figs. 3(a) and (b), demonstrating the reduction in bunch width with increased cooling time.

By fixing the accumulation time to $100 \mathrm{~ms}$, it was also possible to investigate how space-charge effects would affect the bunch width. Figure 4 shows the bunch width characterization of ${ }^{85} \mathrm{Rb}$. In fig. 4(a), the photons arrived with a temporal width of $17 \mu \mathrm{s}$, whereas in fig. 4(b), the bunch width was found to be narrower, $5 \mu \mathrm{s}$ (FWHM).

In fig. $4(\mathrm{a}), 1.2 \times 10^{6}$ ions of ${ }^{85} \mathrm{Rb}$ were observed in the bunch, whereas $5 \times 10^{4}$ ions were observed in fig. 4(b). Therefore, there is a clear broadening of the bunches caused by an increased load into the trap.

At the end of the tests, the effect of the gas pressure on the bunch widths was briefly investigated. Figure 5 shows the difference between the photon arrival times for ${ }^{85} \mathrm{Rb}$ ions, accumulated for $10 \mathrm{~ms}$. The effect of decreasing the gas pressure did not alter the bunch width. However, for the case of cooling with less gas injected, a later arrival of the ion bunch was observed.

\section{Background suppression}

In order to illustrate the effect of bunching the beam for collinear laser measurements on radioactive beams, fig. 6 compares the scanned hyperfine spectra of ${ }^{46} \mathrm{~K}$ for ungated 


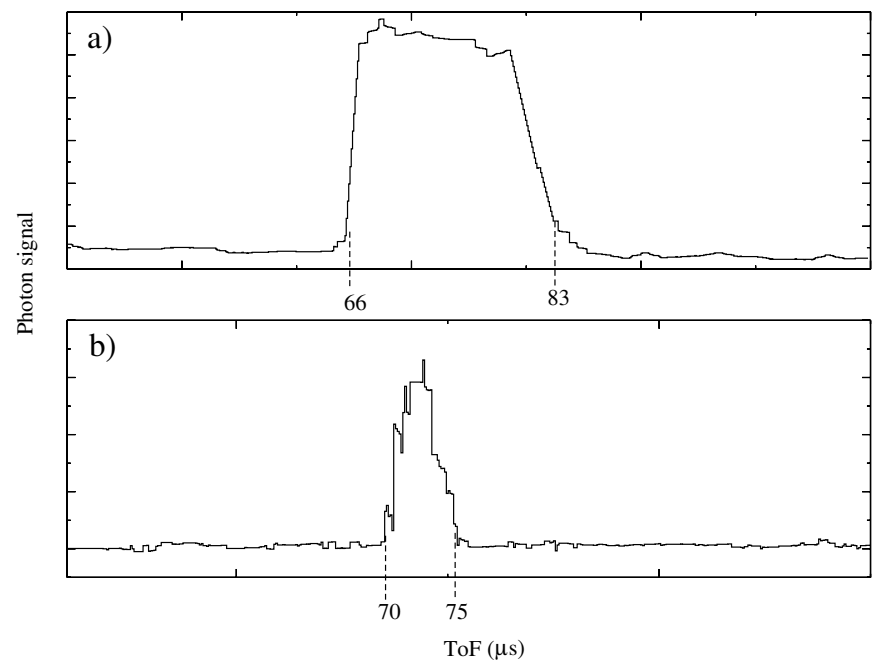

Fig. 4. Bunch width of ${ }^{85} \mathrm{Rb}$. The ions were accumulated for $100 \mathrm{~ms}$. The bunch is three times wider in (a) than in (b) (which is fig. $3(\mathrm{~d})$ on expanded scale), for $1.2 \times 10^{6}$ and $5 \times 10^{4}$ ions in the bunch.

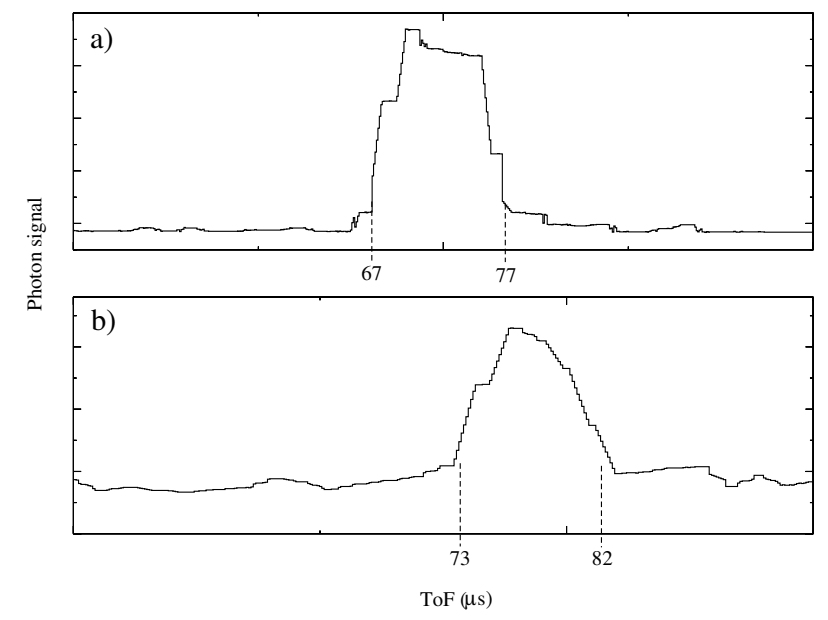

Fig. 5. Bunch width of $1.6 \times 10^{4}{ }^{85} \mathrm{Rb}$ ions. In (a) the gas pressure is 0.1 mbar, whereas in (b), the pressure was reduced to 0.05 mbar.

and gated photons. The data obtained was the result of 22 scans of 201 channels with a dwell time of $0.3 \mathrm{~s}$ for each channel.

Figure 6(b) shows the ungated photons recorded by the data acquisition, which was dominated by $\sim 75000 \mathrm{~s}^{-1}$ due to dark counts (for this measurement the photomultiplier tube was not cooled), and $\sim 2000 \mathrm{~s}^{-1}$ counts due to laser scatter. Later in the experiment, a chiller unit was installed for the photomultiplier tube and the dark count rate was reduced to $150 \mathrm{~s}^{-1}$. Since the ions were accumulated in the trap for $t_{a c c}$ and $t_{w}$ was the bunch width determined from the photon arrival times, the effective measurement time in a given channel was a fraction $t_{w} / t_{a c c}$ of the dwell time. Therefore, the data acquisition was able to register resonant photons, with a background suppression equal to $\frac{t_{a c c}}{t_{w}}$ when compared with the ungated events. It is important to stress the fact that this technique does

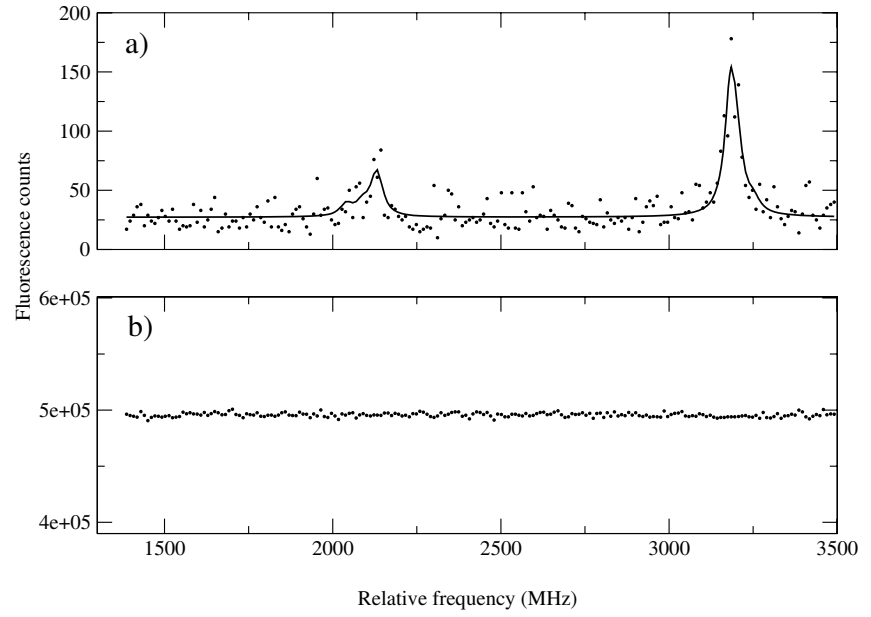

Fig. 6. Resonance spectra of $10^{5}$ ions/s of ${ }^{46} \mathrm{~K}$. After neutralization, the laser excited the $\mathrm{D}_{2}$ line at $766 \mathrm{~nm}$. In (a) the hyperfine structure for gated photons, and (b) ungated. The ions were accumulated for $300 \mathrm{~ms}$ and the gate width was $12 \mu \mathrm{s}$. The laser power was $2 \mathrm{~mW}$. The total scanning time was $22 \mathrm{~min}$.

not suppress beam-related light. With a gate of $12 \mu$ s set in coincidence with the PMT, it was possible to extract the hyperfine structure with a background reduced by a factor of $2.5 \times 10^{4}$, as shown by fig. 6 (a).

\section{Outlook and future prospects}

The result of the tests presented in this work constituted a first demonstration of bunched-beam spectroscopy at CERN-ISOLDE. This technique has paved the way for performing optical measurements on low flux ion beams, which otherwise would be impossible using collinear laser techniques with traditional light detection schemes. The first on-line experiment using cooled and bunched beams at ISOLDE run successfully in July 2008 on neutronrich gallium isotopes. The programme set to measure the neutron-rich copper isotopes ran subsequently in the summer 2008. The spectra obtained from these two experiments are under analysis and will allow ground-state and isomeric spins and moments to be determined. For the future, the investigation on the neutron-rich gallium will be completed in 2009 and it is planned that the gallium programme will be extended to the neutron-deficient gallium isotopes.

The authors would like to thank the AB/ATB and the AB/OP group for their technical support in the operation of ISOLDE and ISCOOL. We also acknowledge the financial support of the European Community under the FP6 "Research Infrastructure Action - Structuring the European Research Area" EURISOL DS Project Contract no. 515768 RIDS.

\section{References}

1. E.W. Otten, Treatise Heavy-Ion Sci. 8, 515 (1989).

2. J. Billowes, P. Campbell, J. Phys. G 469, 707 (1995). 
3. H.-J. Kluge, W. Nörtershäuser, Spectrochim. Acta B 58, 1031 (2003).

4. A. Nieminen et al., Phys. Rev. Lett. 88, 094801 (2002).

5. F. Herfurth et al., Nucl. Instrum. Methods A 469, 254 (2001).

6. A. Nieminen et al., Nucl. Instrum. Methods A 469, 244 (2001).

7. G. Bollen et al., Nucl. Instrum. Methods A 532, 203 (2004).
8. A. Jokinen et al., Nucl. Instrum. Methods B 204, 86 (2003).

9. I. Podadera et al., Nucl. Phys. A 746, 647 (2004).

10. H. Franberg et al., Nucl. Instrum. Methods B 266, 4502 (2008).

11. A.C. Mueller et al., Nucl. Phys. A 403, 234 (1983).

12. R. Neugart, Hyperfine Interact. 24, 159 (1985).

13. W. Geithner et al., Hyperfine Interact. 129, 271 (2000). 